\title{
Age and Gender Association with Post-Operative Respiratory Failure
}

\author{
Singh, Punit ${ }^{1}$, Seifi, Ali $^{2 *}$ \\ ${ }^{I}$ Department of Anesthesiology, University of Texas Health Science Center at San Antonio, \\ San Antonio, TX \\ ${ }^{2}$ Department of Neurosurgery, Division of Neuro Critical Care, University of Texas Health \\ Science Center at San Antonio, San Antonio, TX
}

*Corresponding Author: Seifi, Ali, Department of Neurosurgery, Division of Neuro Critical Care, University of Texas Health Science Center at San Antonio, San Antonio, Texas, Email: seifi@ uthsca.edu

\begin{abstract}
Aims of Study: Postoperative respiratory failure (PORF) is a patient safety indicator (PSI) representing a universal complication for all surgical procedures. This study examined the association between age and gender and the rates of PORF from 2000 to 2012.
\end{abstract}

Methods and Material: We performed a retrospective cohort study on PORF incidence from 2000 to 2012 using the Healthcare Cost and Utilization Project.

Statistical analysis used: Pairwise t tests were performed. P values were calculated, using a Bonferroniadjusted $\alpha$ threshold, to examine PORF differences by age and gender.

Results: Our study revealed that the incidence of PORF had overall significantly decreased from 2000 to $2012(P<0.001)$. The incidence of PORF demonstrated a significant positive correlation with age, with the incidence being high for ages 65 and older $(P<0.001)$. Males had significantly greater PORF incidence rates compared to females in every year of the study period $(P<0.001)$.

Conclusions: Contrary to our results, the current literature on PORF reports a largely increased incidence of PORF, likely due to study specific methodology and definition of PORF. However our study further corroborates the findings of previous studies that age and male gender are associated with increased risk of post-operative respiratory failure in patients undergoing elective surgery.

Keywords: Age; Gender; General anesthesia; Patient safety indicator; Post-operative; Respiratory failure

Key Messages: This study further corroborates the findings of previous studies that age and male gender are associated with increased risk of post-operative respiratory failure in patients undergoing elective surgery.

\section{INTRODUCTION}

Respiratory failure in the post-operative setting is a clinically significant adverse event that leads to increased morbidity, mortality, cost of treatment, and length of hospital stay among different surgical populations. ${ }^{[1,2]}$ Although it has been described in the literature, there remains a lack of information on its epidemiological aspects that may be useful in risk-stratifications and clinical decision-making. The purpose of this study was to report the incidence and examine the association between gender and age and the rates of post-operative respiratory failure (PORF) from 2000 to 2012.

\section{SubJECTS AND METHODS}

We performed a retrospective cohort study on PORF from 2000 to 2012 using Patient Safety Indicators (PSIs) of the Healthcare Cost and Utilization Project (HCUP) that is founded by the Agency for Healthcare Research and Quality (AHRQ) ${ }^{[3]}$ PSIs were developed to provide information to hospitals and clinicians regarding identifying risk factors and potentially preventable adverse events. The PORF PSI was defined as any patient who had: an ICD-9 code for acute respiratory failure in any secondary diagnosis field or mechanical ventilation for 96 consecutive hours or more beginning the day of 
or any day after the operating room procedure, mechanical ventilation for less than 96 hours beginning at least two days after the operating room procedure, or re-intubation the day after surgery or any day thereafter. The specific inclusion and exclusion criterion are provided on the HCUP website. ${ }^{[4]}$ The database included 483,309,639 inpatient admissions for elective surgeries during the 2000-2012 period. Z-statistics were used to assess the significance of group contrasts on proportions. All statistical testing was 2-sided with a nominal significance level of 5\% and p-values were corrected for multiple comparisons using the Bonferroni method.

\section{ReSUlts}

Our analysis revealed that during the thirteenyear study period the incidence of postoperative respiratory failure has decreased from 7.395 in year 2000 to 6.852 in 2012 (P value < $0.001)$, with rates per 1000 elective surgical admissions fluctuating between 6 and 9 each year with the highest rate of 8.959 in 2003 . No trend persisted over time from 2000 to 2012 and no clinically significant correlation appeared to exist however the overall incidence significantly decreased at the end of cohort compared to the beginning $(\mathrm{P}<0.001)$.

When broken down by age, the incidence of post-operative respiratory failure demonstrated a statistically significant positive correlation, with the incidence being the highest for ages 65 and older and the least for ages 18-44 years old $(\mathrm{P}<$ 0.001). Among the geriatric population (age $>65$ years), those aged $>85$ years had the highest incidence, with rates as high as 19 per 1000 elective surgical admissions $(\mathrm{P}<0.001)$. When evaluating the cohort for gender, male patients were shown to have significantly increased rates as compared to female patients $(\mathrm{p}<0.001)$. In fact, the rates of post-operative respiratory failure for men exceeded those for women in all 13 years of the study, sometimes by nearly a twofold difference. On average, men experienced postoperative respiratory failure at a rate of approximately 10 cases per 1000 elective surgical admissions, while women experienced it at an average rate of approximately 6 cases per 1000 elective surgical admissions.

\section{DISCUSSION}

Contrary to our findings, the current literature has largely demonstrated a greater incidence of post-operative respiratory failure, anywhere from 2-20\%. ${ }^{[1,2,5]}$ Reasons for these discrepancies likely arise from differences in study methodology, patient population, and definition of postoperative respiratory failure. Regarding risk factors for PORF, those previously described have been divided into modifiable and non-modifiable risk factors. ${ }^{[1,5,6]}$ Modifiable risk factors are those related to both the patient and the procedure while non-modifiable risk factors are those including gender, age, pre-existing health conditions, anatomic location of surgery, emergency surgery, impaired sensorium (e.g. elderly patients with dementia). ${ }^{[1,7,8]}$ The precise reasons for the associations between postoperative respiratory failure and advancing age and male gender are unknown but the correlation with advancing age is likely due to age-related declines in organ reserve function. Additionally, geriatric patients have difficulty with access to care and therefore a lower preoperative health. The association with male gender may be related to gender-specific differences in the epidemiology of smoking and alcohol use or perhaps other medical conditions not precisely identified.

The major strength of this study comes from the use of the robust HCUP database, thus giving it the statistical power to detect even small differences. Another strength of this study is that it did not focus on a specific patient population or surgical procedure, which enhances the generalizability of the findings.

However, this may also be considered a limitation in that it may not be as applicable to a specific patient with known demographic characteristics or a planned high-risk surgical procedure. The data provided to us by HCUP does not index to type of surgery, which is known to influence the rate of PORF. For example, the incidence of PORF is thought to be high in upper abdominal surgery compared to lower abdominal surgery. ${ }^{[9]}$ The type of surgery may certainly confound the results. Additionally information such as the ASA physical status classification score of patients was unavailable to us, which may be useful to perform further risk stratification. Other researchers have suggested the possibility to predict postoperative respiratory failure based on ASA scores. ${ }^{[10,11]}$

Another limitation of this study is that HCUP uses a slightly different definition of PORF than other authors have reported in the literature, which makes direct comparisons from one study to another difficult. HCUP's 
definition of respiratory failure (see Materials and Methods above) was somewhat more broad than that used by other studies. ${ }^{[2,5,7,12,13]}$ This gives the current study a higher sensitivity but lower specificity than others and may account for some of the variation in our results compared to other investigators. Furthermore, the HCUP software adjusts data for a number of possible confounding variables, however the potential for bias still exists. This is because there are a number of other confounders that were not adjusted for because they were not included in the administrative data transferred from participating hospitals to AHRQ for inclusion in the HCUP database (i.e. smoking status, medical comorbidities).

Finally, any analysis of data is only as reliable as the data itself. There have been recent evaluations of the validity of the postoperative respiratory failure PSI due to discrepancies in ICD-9-CM codes used by medical coders to generate the data used by HCUP. ${ }^{[14,15]}$ Notably, as a result of these studies, the ICD-9$\mathrm{CM}$ codes had been updated in such a way as to enhance the specificity of the PORF without compromising its sensitivity and these changes were carried forward into the ICD-10CM codes. ${ }^{[16]}$

\section{CONCLUSION}

Our study further corroborates the findings of previous studies that age and male gender are associated with increased risk of post-operative respiratory failure in patients undergoing elective surgery. Further studies however, are required to elucidate the precise reasons why many of these associations exist.

\section{REFERENCES}

[1] Sabate S, Mazo V, Canet J. Predicting postoperative pulmonary complications: implications for outcomes and costs. CurrOpinAnaesthesiol. 2014; 27:201-209.

[2] Johnson RG, Arozullah AM, Neumayer L, Henderson WG, Hosokawa P, Khuri SF. Multivariable Predictors of Postoperative Respiratory Failure after General and Vascular Surgery: Results from the Patient Safety in Surgery Study. J Am Coll Surg. 2007; 204:1188-1198.

[3] Healthcare Cost and Utilization Project (HCUP). August 2014. Agency for Healthcare Research and Quality, Rockville, MD. http://www.ahrq.gov/research/data/hcup/index. html PSI \#11: Postoperative Respiratory
Failure. Available at: https://www.quality indicators.ahrq.gov/Downloads/Modules/PSI/V 45/TechSpecs/PSI\%2011\%20Postoperative\%2 0Respiratory\%20Failure\%20Rate.pdf.

[4] Canet J, Gallart L, Gomar C, Paluzie G, Valles J, Castillo J, et al. Prediction of Postoperative Pulmonary Complications in a Populationbased Surgical Cohort. Anesthesiology. 2010;113:1338-1350

[5] Grosse-Sundrup M, Henneman JP, Sandberg WS, Bateman BT, Uribe JV, Nguyen NT, et al. Intermediate acting non-depolarizing neuromuscular blocking agents and risk of postoperative respiratory complications: prospective propensity score matched cohort study. BMJ. 2012; 345.

[6] Masoomi H, Reavis KM, Smith BR, Kim H, Stamos MJ, Nguyen NT. Risk factors for acute respiratory failure in bariatric surgery: data from the Nationwide Inpatient Sample, 20062008. Surgery for Obesity and Related Diseases.2013; 9:277-283.

[7] Rock P, Rich PB. Postoperative Pulmonary Complications. CurrOpinAnaesthesiology. 2003;16:123-132

[8] Fischer JP, Wes AM, Wink JD, Nelson JA, Braslow BM, Kovach SJ. Analysis of Risk Factors, Morbidity, and Cost Associated with Respiratory Complications following Abdominal Wall Reconstruction Journal of Plastic and Reconstructive Surgery. 2014, 133; $1: 147-156$.

[9] Gupta H, Gupta PK, Fang X, Miller WJ, Cemaj $\mathrm{S}$, Forse RA, et al. Development and validation of a risk calculator predicting postoperative respiratory failure. Chest. 2011; 140:12071215. doi: 10.1378/chest.11-0466.

[10] Gupta PK, Gupta H, Sundaram A, Kaushik M, Fang X, Miller WJ, et al. Development and validation of a risk calculator for prediction of cardiac risk after surgery. Circulation 2011; 124:381-7.

[11] Arozullah AM, Daley J, Henderson WG, Khuri SF. Multifactorial Risk Index for Predicting Postoperative Respiratory Failure in Men After Major Noncardiac Surgery. Annals of Surgery, 2000; 232:2; 242-253.

[12] Canver CC, Chanda J. Intraoperative and Postoperative Risk Factors for Respiratory Failure after Coronary Bypass. Ann Thorac Surg. 2003; 75:853-858.

[13] Borzecki AM Kaafarani HM, Utter GH, Romano PS, Shin MH, Chen Q, et al. How Valid is the AHRQ Patient Safety Indicator "Postoperative Respiratory Failure"? J Am Coll Surg. 2011; 212:935-945. 
[14] Utter GH, Cuny J, Sama P, Silver MR, Zrelak PA, Baron R, et al. Detection of Postoperative Respiratory Failure: How Predictive is the Agency for Healthcare Research and Quality's Patient Safety Indicator? J Am Coll Surg. 2010; 211:347-354.
[15] Utter GH, Cuny J, Strater A, Silver MR, Hossli S, Romano PS. Variation in Academic Medical Centers' Coding Practices for Postoperative Respiratory Complications, Implications for the AHRQ Postoperative Respiratory Failure Patient Safety Indicator. Med Care. 2012; 50:792-800.

Citation: Singh, Punit, Seifi, Ali. Age and Gender Association with Post-Operative Respiratory Failure.ARC Journal of Anesthesiology. 2018; 3(2):10-13. DOI: dx.doi.org/10.20431/2455-9792.0302004.

Copyright: (C) 2018 Authors. This is an open-access article distributed under the terms of the Creative Commons Attribution License, which permits unrestricted use, distribution, and reproduction in any medium, provided the original author and source are credited. 